\title{
Takayasu Arteritis Presenting as Severe Pulmonary Hypertension
}

\author{
Rajeev Bhardwaj, ${ }^{1}$ Ranjana Gupta, ${ }^{2}$ Sivaji Patibandla, ${ }^{3}$ Himanshu Tanwar, ${ }^{3}$ Mehroj Ahmed, ${ }^{3}$ Gaurav Aggrawal $^{3}$ \\ 1 Prof. \& Head \\ ${ }^{2}$ Associate Prof \\ ${ }^{3}$ Senior Resident \\ Department of Cardiology MM Institute of Medical Sciences and Research, Mullana, Ambala (India)133207.
}

Address for correspondence: Dr Rajeev Bhardwaj, Prof. \& Head, Cardiology, MMIMSR, Mullana, Ambala (India) 133207.

E mail: rajeevbhardwaj_dr@yahoo.com

\section{ABSTRACT}

Takayasu arteritis is an inflammatory disease of large- and medium-sized arteries, with a predilection for the aorta and its branches. Advanced lesions demonstrate a panarteritis with intimal proliferation. It can affect the aorta, subclavian artery, renal artery, iliac artery, coronary artery, and other blood vessels. Pulmonary arteritis is very uncommon in TA. We describe a case of TA, who primarily presented with pulmonary hypertension.

Key words: Pulmonary arteritis, pulmonary hypertension, Vacuities

\section{CASE}

28 years female presented with dyspnoea for 4 years. It was gradual in onset, slowly progressive. At present she had dyspnoea on routine activities. Patient also had fatigue on exertion. There was no history of orthopnea or paroxysmal nocturnal dyspnoea.

Patient started having swelling of both lower limbs for last two years, which also progressed gradually. Swelling used to subside during sleep. There was no history of $(\mathrm{H} / \mathrm{O})$ periorbital puffiness, jaundice, rash, joint pain, palpitation or abdominal distension, claudication of limbs, exertional presyncope or syncope. Patient had 3 children, all vaginal deliveries. Last child birth was four and half years back. There were no symptoms during pregnancy. Patient took treatment from various hospitals with diagnosis of primary pulmonary hypertension (PPH).

On examination: Right upper limb pulses, and both carotids were impalpable. BP in left upper limb was $110 / 70 \mathrm{mmHg}$, pulse was 96/min, JVP was $12 \mathrm{~cm}$ above the sterna angle, with prominent $\mathrm{V}$ wave and sharp $\mathrm{Y}$ collapse. There was bilateral pedal oedema.

CVS examination: Apex beat was not palpable, grade III parasternal heave was present, right heart border was $3 \mathrm{~cm}$ from the right sterna border. First heart sound was normal, 2nd heart sound showed narrow split with loud pulmonary component. There was pan systolic murmur in tricuspid area, which increased on inspiration.
Investigations:

$\mathrm{Hb} 13.6 \mathrm{gm} \%$

TLC 8.2X 1000/cubic mm (CMM)

Serum bilirubin $0.92 \mathrm{mg} \%$

AST $27 \mathrm{U} / \mathrm{L}$

ALT 29U/L

Platelet count 69X1000/CMM

ECG: QRS axis 110 degree, right ventricular (RV) hypertrophy and $\mathrm{P}$ pulmonale.

X ray chest: Cardiomegaly with CT ratio $-60 \%$ with Right ventricular enlargement, prominent main pulmonary artery and Right atrial (RA) enlargement.

Ultrasound of abdomen: Mild hepatomegaly.

Echocardiography: Left ventricle (LV) was normal in size, showed good contraction with Ejection fraction 65\%. RA and $\mathrm{RV}$ were enlarged, RV free wall contractility was decreased. TAPSE was $11 \mathrm{~mm}$. MPA was dilated. There was severe tricuspid regurgitation with gradient of $65 \mathrm{~mm} \mathrm{Hg}$.

Cardiac catheterization: RV systolic pressure was $88 \mathrm{mmHg}$, and end diastolic pressure $20 \mathrm{~mm} \mathrm{Hg}$. Pulmonary artery pressure was $87 / 29 / 52 \mathrm{mmHg}$. Pulmonary arteriography showed dilated main pulmonary artery (MPA) with small right pulmonary artery (RPA). Oxymetry showed no evidence of shunt. On aortic arch angiography (Figure 1), innominate artery was diffusely diseased, right common carotid was thread like and then showed total occlusion, right subclavian artery was occluded at origin, left common carotid artery showed total occlusion at origin. Left subclavian artery was normal and left vertebral was dilated and supplied whole of brain through collaterals.

Since findings favoured Takayasu arteritis, CT angiography of chest was done to see for evidence of pulmonary arteritis. There was mosaic attenuation of bilateral lungs with areas of fibrosis and honey combing in right lower lobe. There was evidence of severe attenuation of right interlobar, middle and lower lobe branches of RPA, with prominent collateral supply from right bronchial artery (Figure 2). 
Figure 1: Aortic arch angiography shows markedly stenotic innominate artery. Right common carotid artey is thread like at origin and the shows total occlusion. Right subclavian artery shows total occlusion at origin. Left common artery shows total occlusion at origin. Left subclavian artery is normal.

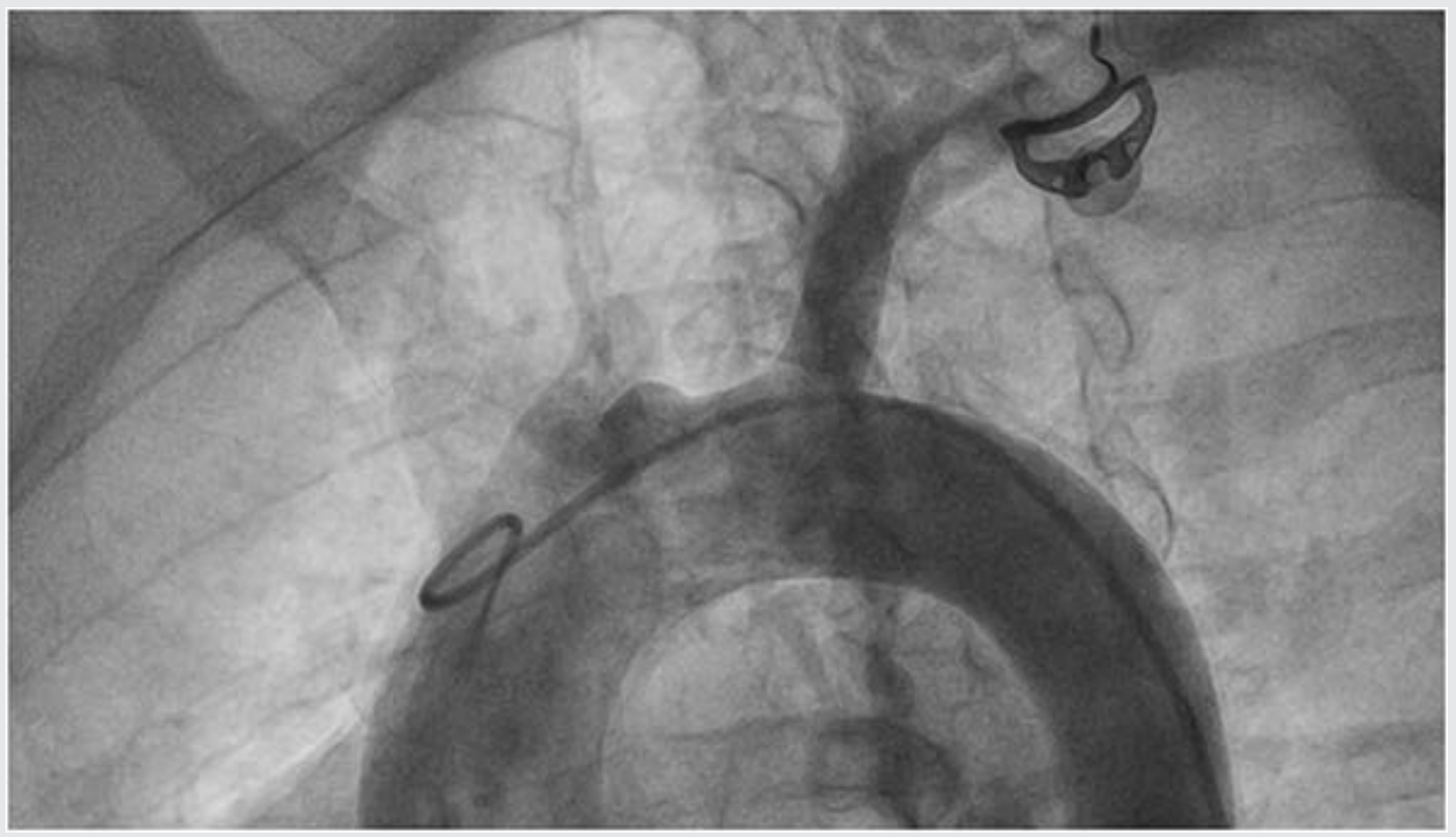

Figure 2: CT angiography of chest show evidence of severe attenuation of right interlobar, middle and lower lobe branches of right pulmonary artery.
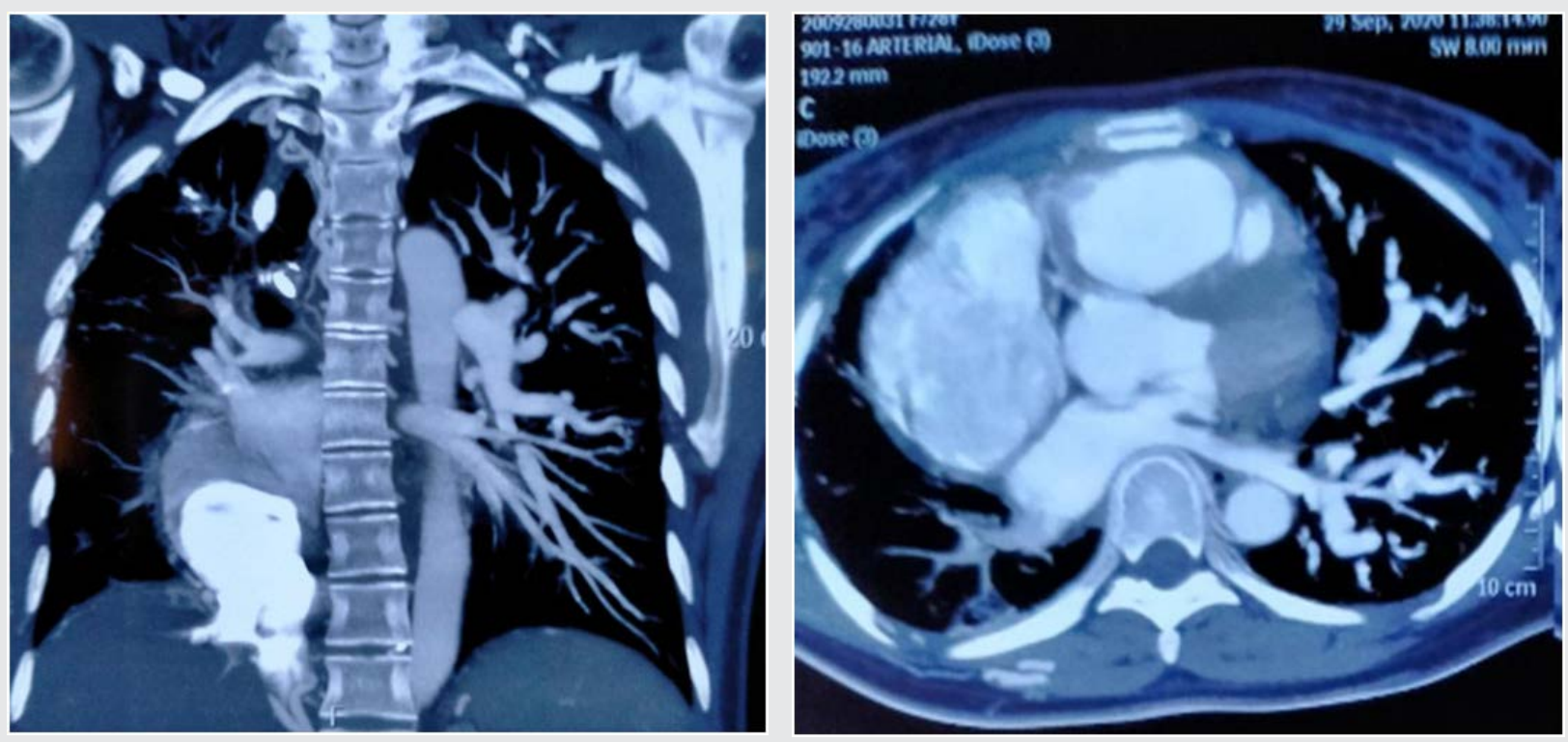


\section{DISCUSSION}

Takayasu's arteritis (TA) is a form of chronic vasculitis primarily involving the large arteries and their main branches, and the pathological manifestation is arterial full-thickness inflammation. ${ }^{1}$ TA can affect the aorta, subclavian artery, renal artery, iliac artery, coronary artery, and other blood vessels. The clinical manifestations vary greatly depending on the area, severity, and duration of vascular involvement. TA usually progresses through three stages: ${ }^{2}$ an early stage characterized by nonspecific symptoms, a vasculitis stage characterized by a systemic inflammatory response and thickening of the vascular walls, and a static stage characterized by dissipating inflammation and occlusion of affected vessels. Notably, the vasculitis stage is a crucial period for diagnosis and treatment.

Takayasu arteritis can be divided into the following six types based on angiographic involvement. ${ }^{3}$

Type I - Branches of the aortic arch

Type Ila - Ascending aorta, aortic arch, and its branches Type IIb - Type Ila region plus thoracic descending aorta Type III - Thoracic descending aorta, abdominal aorta, renal arteries, or a combination

Type IV - Abdominal aorta, renal arteries, or both

Type $\mathrm{V}$ - Entire aorta and its branches

The presentation of Takayasu arteritis is heterogeneous. Approximately $10 \%$ of patients with Takayasu arteritis are asymptomatic, and the diagnosis is suggested only by abnormal vascular findings on physical examination. ${ }^{4,5}$ Constitutional symptoms may precede clinical vascular involvement.

Constitutional symptoms include the following

- Headache

- Malaise

- Arthralgias

- Fever

- Weight loss

The Ishikawa criteria (1986) have been useful in defining Takayasu arteritis. One criterion is age younger than 40 years at diagnosis or at onset of characteristic signs and symptoms of 1-month duration.

Two major criteria involve lesions in the left and right mid subclavian artery, with the most severe stenosis or occlusion present in the mid portion of the artery from a $1 \mathrm{~cm}$ point proximal to the left and right, respectively, of the vertebral artery orifices to a 3-cm distal point to the orifice, as determined by angiography.

The minor criteria consist of annuloaortic ectasia or aortic regurgitation on angiography or echocardiography, and lesions of any of the following vessels:

- Pulmonary artery

- Left mid common carotid artery

- Distal brachiocephalic trunk

- Descending aorta

- Abdominal aorta
TA can involve the pulmonary artery. ${ }^{6,7}$ The incidence of pulmonary arteritis (PA) in patients with TA varies greatly among studies (0-56\%), ${ }^{8,9}$ Pulmonary hypertension $(\mathrm{PH})$ occurs in 12 to $13 \%$ of patients with TA and in $42.2 \%$ of patients with PA. ${ }^{6,9,10}$ No large-sample study of PA and $\mathrm{PH}$ in patients with TA has been performed to date. Only one Chinese hospital specializing in cardiovascular diseases reported that the incidence of $\mathrm{PH}$ in patients with TA involving the pulmonary artery reached $78.1 \% .{ }^{11}$ Pulmonary artery involvement in patients with TA increases the likelihood of misdiagnosis or delayed diagnosis because of the nonspecific respiratory manifestations and lack of symptoms of systemic vessel involvement. $\mathrm{PH}$ is a late manifestation of PA that indicates a weaker response to treatment and a poor prognosis. ${ }^{6}$ Thus, it is vital to achieve an early diagnosis of PA in patients with TA.

Yang et $\mathrm{al}^{12}$ reviewed 815 patients with TA during a 30-year period in a single centre and found that (1) PA was present in $6.3 \%$ of patients with TA and that this proportion increased over time, (2) $58.8 \%(30 / 51)$ of patients with PA developed $\mathrm{PH}$, and (3) $82.4 \%(42 / 51)$ of patients with PA had lung parenchymal lesions on CT scans, representing indirect signs of pulmonary artery involvement. Compared with the $\mathrm{PH}$ group, the non- $\mathrm{PH}$ group had different clinical features and imaging findings and a better prognosis. The main symptoms in this study were dyspnea $(70.6 \%)$, cough $(66.7 \%)$, hemoptysis $(47.1 \%)$, and chest pain $(45.1 \%)$, while $5.9 \%$ of patients had no respiratory symptoms. In this study, the median time from the initial symptoms to definitive diagnosis was $13.5(1-186)$ months. In addition, $80 \%$ of patients in this study were diagnosed with other diseases at their first visit, mostly pulmonary infection, pulmonary tuberculosis, idiopathic $\mathrm{PH}$, and pulmonary embolism.

Patients who have TA with pulmonary artery involvement have occult-onset and nonspecific symptoms, making early diagnosis difficult. Accurate diagnosis of pulmonary artery involvement virtually always depends on imaging studies because the clinical manifestations and laboratory test results are usually nonspecific. Typical CT manifestations of pulmonary artery involvement include artery wall thickening and enhancement in the early disease stages and luminal stenosis or occlusion in the chronic stages. ${ }^{13,14}$

Our patient presented with dyspnoea. He did not have symptoms suggestive of TA and had been diagnosed as a case of PPH. Echocardiography showed evidence of PAH with RV dysfunction. However clinical examination and angiography suggested the diagnosis of TA. CT angiography suggested involvement of lungs and PA. 


\section{REFERENCES}

1. Numano F, Okawara M, Inomata H, Kobayashi Y. Takayasu's arteritis. Lancet. 2000;356(9234):1023-1025.

2. Castaner E, Alguersuari A, Gallardo X, Andreu M, Pallardo Y, Mata JM, et al. When to suspect pulmonary vasculitis: radiologic and clinical clues. Radiographics. 2010;30(1):33-53.

3. Hata A, Noda M, Moriwaki R, Numano F. Angiographic findings of Takayasu arteritis: new classification. Int J Cardiol. 1996 Aug. 54 Suppl:S155-63.

4. Seyahi E. Takayasu arteritis: an update. Curr Opin Rheumatol. 2017 Jan. 29(1):51-56.

5. Maksimowicz-McKinnon K, Clark TM, Hoffman GS. Limitations of therapy and a guarded prognosis in an American cohort of Takayasu arteritis patients. Arthritis Rheum. 2007 Mar. 56(3):1000-9.

6. Toledano K, Guralnik L, Lorber A, Ofer A, Yigla M, Rozin A, et al. Pulmonary arteries involvement in Takayasu's arteritis: two cases and literature review. Semin Arthritis Rheum. 2011;41(3):461-470.

7. Wang X, Dang A, Chen B, Lv N, Liu Q. Takayasu arteritis-associated pulmonary hypertension. J Rheumatol. 2015:42(3):495-503.

8. Hotchi M. Pathological studies on Takayasu arteritis. Heart Vessels Suppl. 1992; 7:11-17.

9. Sharma BK, Jain S, Radotra BD. An autopsy study of Takayasu arteritis in India. Int J Cardiol. 1998;66:S85-S90.

10. Bicakcigil M, Aksu K, Kamali S, Ozbalkan Z, Ates A, Karadag O, et al. Takayasu's arteritis in Turkey - clinical and angiographic features of 248 patients. Clin Exp Rheumatol. 2009;27(1 Suppl 52):S59-S64.

11. Changming $X$, Zhihong L, Jianguo $H$. Xinhai N, Xiansheng $C$. Clinical analysis of pulmonary Vasculitis in 41 patients. Chin Circ J. 2010;25(1):4446.

12. Jing Yang, Min Peng, Juhong Shi Wenjie Zheng, and Xuezhong Yu. Pulmonary artery involvement in Takayasu's arteritis: diagnosis before pulmonary hypertension. BMC Pulm Med. 2019; 19: 225.

13. Park JH, Chung JW, Im JG, Kim SK, Park YB, Han MC. Takayasu arteritis: evaluation of mural changes in the aorta and pulmonary artery with $\mathrm{CT}$ angiography. Radiology. 1995;196(1):89-93.

14. Castaner E, Alguersuari A, Andreu M, Gallardo X, Spinu C, Mata JM. Imaging findings in pulmonary vasculitis. Semin Ultrasound CT MR. 2012;33(6):567-579. 